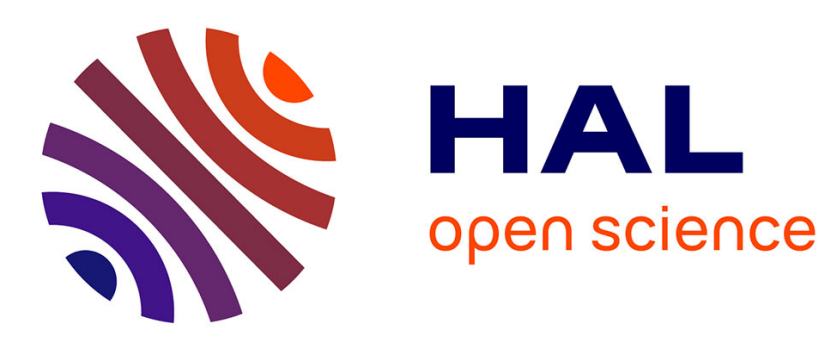

\title{
Sensor network design for failure detection and isolation
}

Marie Luong, Didier Maquin, José Ragot

\section{To cite this version:}

Marie Luong, Didier Maquin, José Ragot. Sensor network design for failure detection and isolation. IFAC Conference on Control of Industrial Systems, May 1997, Belfort, France. 6 p. hal-00311228

\section{HAL Id: hal-00311228 \\ https://hal.science/hal-00311228}

Submitted on 13 Aug 2008

HAL is a multi-disciplinary open access archive for the deposit and dissemination of scientific research documents, whether they are published or not. The documents may come from teaching and research institutions in France or abroad, or from public or private research centers.
L'archive ouverte pluridisciplinaire HAL, est destinée au dépôt et à la diffusion de documents scientifiques de niveau recherche, publiés ou non, émanant des établissements d'enseignement et de recherche français ou étrangers, des laboratoires publics ou privés. 


\title{
SENSOR NETWORK DESIGN FOR FAILURE DETECTION AND ISOLATION
}

\author{
Marie Luong, Didier Maquin and José Ragot \\ Centre de Recherche en Automatique de Nancy - CNRS UA 821 \\ Institut National Polytechnique de Lorraine \\ 2, Avenue de la Forêt de Haye - 54516 Vandoeuvre Cedex \\ Phone: +(33) 383595683 Fax: +(33) 383595644 \\ Email: \{mluong, dmaquin, jragot\}@ensem.u-nancy.fr
}

\begin{abstract}
The model-based approach to fault detection and isolation in automated process has received considerable attention during the last two decades. One of the approaches to facilitate fault isolation is to design a structured residual set. The term structured here means that each residual is designed to be sensitive to a certain group of faults and insensitive to others. However, despite this issue has been recognised for a long time, the problem of designing such residuals is not completely solved. In the context of linear static systems, this paper proposes a new method for sensor network design respecting some sensor failure detectability and isolability requirements.
\end{abstract}

Key Words: sensor network design, redundancy, failure detection and isolation, modelbased diagnosis, linear systems, carriage networks.

\section{INTRODUCTION}

In all industrial processes, measurements of the process states are made during operation for the purpose of control and supervision. The performances of the latter depend on extensive and accurate process data which are obtained through these measurements. It is never possible to measure each and every process variable, however, the exploitation of mass and energy balance relationships between different variables of a steady-state process allows some or all of the unmeasured variables to be estimated. Most of the decisions regarding the selection of variables to be measured and the corresponding sensors to be used are made early in the design phase of a plant. This problem is referred to as the sensor network design problem.

Traditionally, the selection of sensors was driven by the needs of the control loop design. However, emerging data reconciliation techniques have set up the scenario for a revision of the criteria of sensor location. Indeed, different criteria have been used for the design, such as ensuring observability of variables (Vaclavek and Loucka, 1976), maximising the accuracy of estimation of variables (Kretsovalis and Mah, 1987; Bagajewicz, 1995), minimisation of cost while ensuring the observability of variables (Madron and Veverka, 1993), maximising the reliability of estimation of variables when sensor failures are likely to occur (Ali and Narasimhan, 1993), minimise the cost or maximise the reliability of the instrumentation system while satisfying constraints on redundancy degrees of variables (Maquin et al., 1996).

In this paper, we investigate the problem of sensor network design for linear processes in order to guarantee the detectability and/or the isolability of sensor failures. An attempt to solve this type of problem has been published very recently, in a slight different context (Carpentier and Litwak, 1996). The proposed design is based on extensive usage of the concept of redundancy degree of a variable (Maquin et al., 1996) and structural analysis of the system model. As the constraints of detectability and isolability are expressed in terms of redundancy degree constraints, they may be taken into account within other types of constraints. So, the sensor network design, leads to minimise the cost of sensor installation while satisfying some constraints such the unmeasurability of certain variables, the obligation of 
measurement of other ones (or consideration of already measured variables), the variable observability or the insurance of a certain redundancy degree. The problem may be solved either with a matricial analysis of the cycle matrix associated to the graph representing the linear process, or using technique of mixed linear programming.

This paper is organised as follows. In the second section, the process codification is described Then, the main results of redundancy analysis are described in the third section. In the fourth section, the redundancy degree of a variable is defined and methods of evaluation of this degree are presented. The fifth section is dedicated to failure detection and isolation. These properties are remembered using the occurrence matrix of redundancy equations and the failure signature. The links between failure detectability, isolability and redundancy degree of a variable are pointed out in the sixth section. A method for designing sensor network satisfying failure detectability and isolability requirements is then described in the following section. The problem is formulated as a linear programming problem. The resulting optimisation problem is solved using a binary mixed integer programming method in order to take into account the occurrences and locations of sensors. The last section presents a sensor network design example.

\section{PROCESS CODIFICATION}

We consider only processes which may be described by a carriage network (figure 1), the nodes of which correspond to elementary equations and the arcs to variables. For a physical interpretation, a node may be a processing unit and an arc, a material or energy transfer stream.

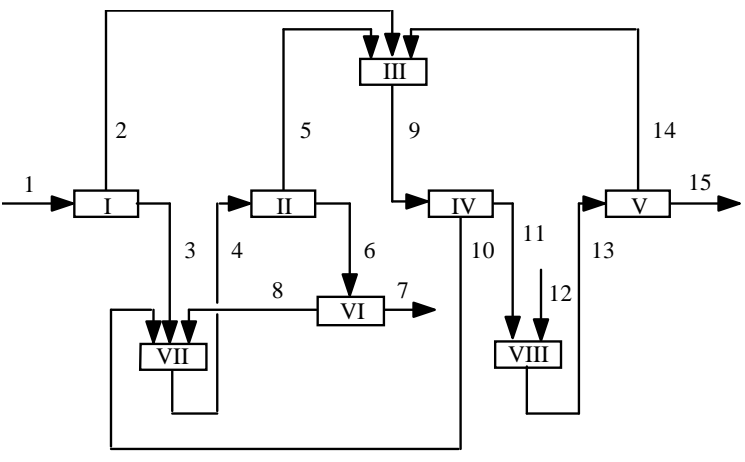

Figure 1: a carriage network

This type of process may be described by a set of linear equations:

$A X^{*}=0$

where $A(n, v)$ is the so-called node incidence matrix with $n$ giving the number of equations and $v$ the number of variables and $X^{*}(v, 1)$ is the variable vector. In a certain number of applications, it is much easier to use the cycle matrix of the associated graph. In order to transform the network into a graph, all the input/output arcs are linked to a so-called environment node.

The matrix $B$ of the fundamental cycles of the graph may be easily deduced from the incidence matrix (Deo, 1967). Using linear combinations of the rows of this fundamental cycle matrix, it is possible to generate the matrix $C_{t}$ of all the cycles of the graph.

\section{REDUNDANCY ANALYSIS}

Let us first recall the description of the considered systems, in the failure-free case:

model equation: $\quad A X^{*}=0$

measurement equation: $X_{m}=H X^{*}+\varepsilon$

where $X_{m}(m, 1)$ is the vector of measurements, $H$ $(m, v)$ the measurement selection matrix (as all process variables are not measured) and $\varepsilon$ is a vector of measurement errors which are normally distributed with zero mean and known variance matrix $V$.

Assuming that all the measurements are direct, the $X^{*}$ vector of variables can be splited into measured variable vector $X_{m}^{*}(m, 1)$ and unmeasured variable vector $X_{\bar{m}}^{*}((v-m), 1)$. Then, the system (2) may be written:

$$
\begin{aligned}
& A_{m} X_{m}^{*}+A_{\bar{m}} X_{\bar{m}}^{*}=0 \\
& X_{m}=X_{m}^{*}+\varepsilon
\end{aligned}
$$

If $\operatorname{rank}\left(A_{\bar{m}}\right)<v-m$, the system is said to be redundant and the redundancy equations may be obtained by projection. Premultiplying equation (3a) by a matrix $Q$ defined by $Q A_{\bar{m}}=0$, we obtain the following equation:

$$
\begin{aligned}
& M_{r} X_{m}^{*}=0 \\
& \text { with } M_{r}=Q A_{m}
\end{aligned}
$$

The matrix $M_{r}$ defines the redundancy equations of the considered system.

\section{DEGREE OF REDUNDANCY CONCEPT}

From the preceding notion of redundancy, one may define the concept of degree of redundancy (Maquin et al., 1996). Let us begin by the notion of minimal observability. A variable is redundant of degree 0 (minimal observability) if there exists, at least, a configuration such that the breakdown of only one sensor makes this variable inaccessible.

This notion may be extended. A redundant variable of degree $k$ is an observable variable which value remains deducible even when $k$ whatever sensors simultaneously breakdown. The determination of the degree of redundancy of a variable is easily obtained 
by applying the following rule which uses the matrix of all the cycles of the associated graph:

A variable is redundant of degree $k$ if, and only if, it belongs only to cycles comprising at least $k+1$ measured variables.

The degree of redundancy of a variable is easily determined by counting the minimum number of measured variables in the cycles where it intervenes.

The redundancy degree of a variable may also be defined from the set of redundancy equations of a system defined in the previous section (matrix $M_{r}$ ). In fact, this set of equations is not unique ; let us call this set primary set of redundancy equations. From this primary set, it is possible to construct a secondary set by generation of all the possible linear combinations of primary set equations. Now, let us consider the whole set of equations (primary plus secondary). Then, the redundancy degree of a variable may be defined as follows. Any variable belonging to whatever redundancy equation is a least redundant of degree 1 moreover if there exists a subset of $k(k \geq 2)$ redundancy equations (among the whole set of redundancy equations as defined previously) with a unique common measured variable, the redundancy degree of this variable is equal to $k$.

\section{FAILURE DETECTION AND ISOLATION}

Process measurements are subject to two main types of errors. The first embraces random errors, generally assumed independent and Gaussian with zero mean. The second embraces gross errors provoked by nonrandom events such as sensor malfunction or instrument biases.

Various methods have been proposed in recent years for gross error detection and isolation in process data (Mah and Tamhane, 1982; Serth and Heenan, 1986; Rosenberg et al., 1987; Narasimhan and Mah, 1987; Crowe, 1989). The parity space or imbalance residual method, which are strictly identical (Maquin and Ragot, 1991), is briefly remember here.

Due to the presence of measurement errors, the measurement vector $X_{m}$ does not satisfy the constraint equations and the residual vector, $R$ is given by:

$$
R=M_{r} X_{m}
$$

With the previous hypothesis of a Gaussian distribution of the measurement errors, the vector $R$ also follows a normal distribution with zero mean and covariance $V_{R}=M_{r} V M_{r}^{T}$. In order to compare the elements of the $R$ vector, let us define a normalised residual vector $R_{N}$ with the entry $R_{N}(i)$ defined by:

$R_{N}(i)=\frac{R(i)}{\sqrt{V_{R}(i, i)}} \quad$ for $i=1, \ldots, r$
Each entry $R_{N}(i)$ follows a normal distribution with zero mean and unity variance. A simple statistical two tailed test can therefore be employed: we may concluded that residual $i$ is not a normal one if:

$\left|R_{N}(i)\right|>t$

Classically, one may choose the critical constant $t$ to control the familywise Type I error rate at some preassigned level $\alpha$. Even if we assume the presence of only one gross error, the relationship between the "bad" residual(s) and the suspect measurement is not so easy. It depends on the structure of the equations and the location of the failure. In some cases, it is not possible to suspect only one measurement. In order to locate suspect measurements, each individual test yields a Boolean decision, the full set of parallel tests resulting in a Boolean vector, the failure signature. This signature is then analysed in order to arrive at a failure inference. Let us defined the occurrence matrix as the absolute value of the matrix of redundancy equations. Since each column of this matrix corresponds to a process variable and each row to a residual, a 1 in any position of this matrix signifies that the given residual is influenced by a failure on the given variable. Thus under ideal circumstances, the signature of a failure is identical to the respective column of the occurrence matrix. This implies that for each failure to be detectable, no column of the matrix should contain only zero elements, and for each signature to be isolable, all columns must be different (Gertler and Singer, 1985).

It is important to note that the properties of failure detectability and isolability don't depend on the choice of the primary set of redundancy equations. So, if a failure is detectable or isolable using the failure signature constructed on the basis of the primary set of equations, so it is using the failure signature constructed on the basis of the whole set of redundancy equations as defined in the previous section.

\section{DETECTABILITY, ISOLABILITY AND REDUNDANCY DEGREE}

In this section, the close links between failure detectability, isolability and redundancy degree of a variable are emphasised.

The failure of a sensor is detectable if and only if the corresponding variable is, at least, redundant of degree one. This first result is obvious. For a sensor failure to be detectable, the corresponding variable might intervene in the calculus of, at least, one residual. As residuals are obtained from the redundancy equations, the considered measured variable might belong to a redundancy equation, so might be redundant of degree one.

The second result is more interesting. The failure of a sensor is isolable if and only if the corresponding variable is, at least, redundant of degree two. Let us 
consider two variables $x_{i}$ and $x_{j}$ which degree of redundancy is equal to two. Using the definition of the degree of redundancy given in section 3 , it means that $x_{i}$ occurs in two redundancy equations, let $r_{a}$ and $r_{b}$, of the whole set of redundancy equations such $x_{i}$ is the unique common measured variable. The variable $x_{j}$ also occurs in two such type of redundancy equations. So, the subvector $v_{i}$ of the occurrence matrix corresponding to the two redundancy equations $r_{a}$ and $r_{b}$ for the variable $x_{i}$ is the following:

$v_{i}=r_{a}\left(\begin{array}{l}x_{i} \\ 1 \\ 1\end{array}\right)$

Taking into account the definition of the redundancy degree of a variable, if $x_{j}$ occurs in one of the redundancy equations among $r_{a}$ and $r_{b}$, it cannot occur in the other. Another possibility is that $x_{j}$ does not occur in any of these two equations. So the subvector $v_{j}$ of the occurrence matrix corresponding to the two redundancy equations $r_{a}$ and $r_{b}$ for the variable $x_{j}$ is one of the following:

$v_{j}=r_{r_{b}}\left(\begin{array}{l}1 \\ 0\end{array}\right)$

$$
v_{j}=r_{r_{b}}\left(\begin{array}{l}
x_{j} \\
1
\end{array}\right)
$$$$
v_{j}=r_{r_{b}}\left(\begin{array}{l}
x_{j} \\
0
\end{array}\right)
$$

As the subvectors $v_{i}$ and $v_{j}$ are systematically distinct, and taking into account the invariance of the isolability property with regard to the choice of the primary set of equation, one deduces that the failure of a sensor is isolable if the corresponding variable is, at least, redundant of degree two.

This last result is the main result of the paper as isolability of sensor failures may now be taken into account in sensor network design.

\section{SENSOR NETWORK DESIGN}

Now, we propose a method for designing sensor network satisfying failure detectability and isolability requirements. Taking into account the previous equivalencies, we specify the lists the variables of which we ought to ensure a given degree of redundancy (list $L_{k}$ for the variables which must be redundant of degree $k$ ). Moreover, a weight, proportional to the installation cost of the corresponding sensor, is associated to each variable.

The main goal of the design then consists in determining the variables which must be measured in order to satisfy the constraints on the degrees of redundancy whilst minimising the global cost of installation (if all the costs are identical, it is the number of sensors which is minimised). This problem may be formulated as follows:
Problem $P_{1}\left\{\begin{array}{l}\underset{u_{i}}{\operatorname{minimum}} \sum_{i=1}^{v} w_{i} u_{i} \\ \text { subject to } \partial r\left(x_{j}\right) \geq k \quad \forall x_{j} \in L_{k}\end{array}\right.$

where $w_{i}$ represents the installation cost of the sensor measuring the variable $x_{i}, u_{i}$ ie a Boolean number which indicates if the variable $x_{i}$ must be measured to meet the redundancy constraints (1: must be measured, 0: elsewhere), and $\partial r\left(x_{j}\right)$ denotes the redundancy degree of the variable $x_{j}$. In fact, some components $u_{i}$ are fixed to 1 as all the variables on which properties of sensor failure (detectability or isolability) are imposed must, of course, be measured.

The problem $P_{1}$ may easily be transformed into a linear programming problem. Indeed, sensor network design consists in measuring a given number of variables per cycles depending on the redundancy degree which must be ensured whilst minimising the associated cost. This problem can be stated as follows:

Problem $P_{2}\left\{\begin{array}{l}\underset{U}{\operatorname{minimum}} W^{T} U \\ \text { subject to } C U \geq N\end{array}\right.$

where $W=\left[w_{i}\right]$ is the cost or weight vector of dimension $v, U=\left[u_{i}\right]$ is a partially unknown vector of Boolean numbers. $C$ is a cycle matrix the construction of which will be explained latter and $N$ is a vector which entries are equal to the minimum number of measurements per cycle for satisfying the redundancy constraints.

Let us now explain the construction of $C$ and $N$. The matrix $C$ is built from the matrix of all the cycles of the graph removing the cycles involving only variables which do not belong to any lists $L_{k}$. Indeed, there is not any constraints on the variables belonging to these cycles. The entries of the vector $N$ are computed as follows. If a cycle contains a variable belonging to the list $L_{k}$, the redundancy constraints will be satisfied only if $k+1$ variables belonging to this cycle are measured. As a cycle may contain variables belonging to different lists $L_{k}$, the entry $n_{i}$ will be equal to the maximum number of variables which must be measured. If a given cycle contains less variables than this minimum number of variables to be measured, the problem has no solution, except if we accept to use hardware redundancy, that is to say to place more than one sensor for measuring a variable.

The problem may be simplified when some variables are already measured. In fact, two situations may occur. The first one concerns a complete sensor network design, i.e. there is not any measurements yet. However, taking into account a previous remark, even in this situation, some variables must be measured (those on which it is desired to obtain detectability or isolability of sensor failure). The 
second situation deals with a partial design taking into account a list of already measured variables. In all the cases, the problem of sensor network design must be solved taking into account that some variables must be measured (or are already measured which is equivalent). Therefore, some of the $u_{i}$ are fixed to 1 and it is very useful to take this situation into account before solving the problem. Indeed, the size of the problem (15) may be reduced, if the columns of the cycle matrix $C$ corresponding to the measured variables are cancelled and if, simultaneously, the vector $N$ of the minimum number of measurements per cycle is updated (each entry $n_{i}$ must be decreased of the number of measured variables in the cycle number $i$ ). After this treatment, some cycles of the $C$ matrix don't support any constraints, that is to say $n_{i}=0$ and these cycles and the corresponding entry in the vector $N$ may be removed.

In this way, it is also possible to take into account the unmeasurability of certain variables. Indeed, it suffices to cancel in the $C$ matrix the columns corresponding to these unmeasurable variables without other modifications.

\section{EXAMPLES}

Let us consider the process described by figure 1 . The incidence matrix corresponding to the network is given in table 1 (for convenience, the "." states for the value 0 ), and the associated fundamental cycle matrix is given in table 2 :

\section{Table 1: incidence matrix}

\begin{tabular}{|c|c|c|c|c|c|c|c|c|c|c|c|c|}
\hline & 12 & 3 & 4 & 5 & 6 & 7 & & $\begin{array}{ll}9 & 10\end{array}$ & 11 & 12 & & 415 \\
\hline I & $1-1$ & -1 & . & . & . & . & & . & 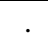 & & & \\
\hline II & . & . & 1 & -1 & & . & & . & . & & & \\
\hline III & 1 & . & . & 1 & . & . & & -1 & . & . & & 1 \\
\hline IV & . & . & . & . & & . & & $1-1$ & -1 & . & & \\
\hline V & . & . & . & . & . & . & & . . & . & & & $-1-1$ \\
\hline VI & . & . & . & . & 1 & $-1-$ & & & . & & & \\
\hline VII & . & 1 & -1 & . & . & . & 1 & . -1 & . & ${ }^{\circ}$ & . & \\
\hline VIII & & . & & . & . & & & .. & 1 & & -1 & \\
\hline
\end{tabular}

$\underline{\text { Table 2: fundamental cycle matrix }}$

\begin{tabular}{|c|ccccccccccccccc|}
\hline & 1 & 2 & 3 & 4 & 5 & 6 & 7 & 8 & 9 & 10 & 11 & 12 & 13 & 14 & 15 \\
\hline 1 &. & 1 & 1 & 1 & 1 &. &. &. &. &. &. &. &. &. &. \\
2 & 1 &. & 1 & 1 &. & 1 & 1 &. &. &. &. &. &. &. &. \\
3 &. &. &. & 1 &. & 1 &. & 1 &. &. &. &. &. &. &. \\
4 &. & 1 & 1 &. &. &. &. &. & 1 & 1 &. &. &. &. &. \\
5 & 1 & 1 &. &. &. &. &. &. & 1 &. & 1 & 1 &. &. &. \\
6 &. &. &. &. &. &. &. &. & 1 &. & 1 &. & 1. & 1 &. \\
7 & 1 & 1 &. &. &. &. &. &. & 1 &. & 1 &. & 1 &. & 1 \\
\hline
\end{tabular}

As the matrix of all the cycles of the graph comprises 127 cycles, it is not shown here. Assume that the vector of measured variables is the following

$X_{m}=(x 2, x 3, x 5, x 7, x 10, x 11, x 14, x 15)^{T}$. Using simple linear combinations of equations (rows) and exchanging of variables (columns) lead to the canonical form of this incidence matrix (table 3 ) where the left column denotes the names of the transformed equations:

Table 3: canonical form of the incidence matrix

\begin{tabular}{|c|cccccccc|c|cccccc|}
\hline & 2 & 3 & 5 & 7 & 10 & 11 & 14 & 15 & 8 & 1 & 4 & 9 & 13 & 6 & 12 \\
\hline $\mathrm{a}$ & -1 &. & -1 &. & 1 & 1 & -1 &. &. &. &. &. &. &. &. \\
$\mathrm{~b}$ &. & 1 & -1 & -1 & 1 &. &. &. &. &. &. &. &. &. &. \\
\hline $\mathrm{c}$ & -1 & -1 &. &. &. &. &. &. &. & 1 &. &. &. &. &. \\
$\mathrm{~d}$ &. &. & -1 & -1 &. &. &. &. & -1 &. & 1 &. &. &. &. \\
$\mathrm{e}$ & -1 &. & -1 &. &. &. & -1 &. &. &. &. & 1 &. &. &. \\
$\mathrm{f}$ &. &. &. &. &. &. & -1 & -1 &. &. &. &. &. &. &. \\
$\mathrm{~g}$ &. &. &. & -1 &. &. &. &. &. &. &. &. & 1 &. &. \\
$\mathrm{~h}$ &. &. &. &. &. &. &. &. & -1 &. &. &. &. & 1 &. \\
\hline
\end{tabular}

For a detailed description of the used transformations which lead to the above form of the incidence matrix, we refer the reader to earlier published works (Maquin et al., 1987; Ragot et al., 1990). This canonical form can be easily read. The measured variables $x 2, x 3, x 5, x 7, x 10, x 11$ and $x 14$ are linked by redundancy equations (defined by row $\mathrm{a}$ and $\mathrm{b}$ of the canonical form). The unmeasured variables $x 1$, $x 9, x 12$ and $x 13$ can be deduced as each one of them only appears as a single unknown in one equation (rows $\mathrm{c}$, e, $\mathrm{f}$ and $\mathrm{h}$ of the canonical form). The measured variable $x 15$ does not appear in any redundancy equation. Finally the unmeasured variables $x 4, x 6$ and $x 8$, which appear in equations containing at least two unknowns, cannot be deduced.

So, for the considered example, the matrix $M_{r}$ of redundancy equations is the following:

\section{Table 4: matrix of redundancy equations}

$$
M_{r}=\begin{array}{cccccccc|}
\hline 2 & 3 & 5 & 7 & 10 & 11 & 14 & 15 \\
\hline-1 & . & -1 & . & 1 & 1 & -1 & . \\
. & 1 & -1 & -1 & 1 & . & . & . \\
\hline
\end{array}
$$

Now, let us consider the following problem:

How many sensors must be added and which variables must be measured in order to be able to detect a sensor failure on variable $x 15$ and to isolate sensor failures on variables $x 5$ and $x 11$ ?

The reader may remark that, with the initial set of measurements, these constraints are not satisfied. Neither sensor failure is isolable and the sensor failure of variable $x 15$ is not detectable as this variable doesn't occur in any redundancy equation. Taking into account the results of section 6 , this problem may be solved by designing a sensor network which guarantee at least a redundancy degree of $x 15$ equal to 1 , and redundancy degrees of $x 5$ and $x 11$ equals to 2, i.e. $L_{1}=\{x 15\}$ and

$L_{2}=\{\mathrm{x} 5, \mathrm{x} 11\}$. In this problem, some variables are already measured and using the treatment prescribed in the previous section, the matrix dimension of $C$ reduces from $(127,15)$ to $(8,7)$. The resulting problem of optimisation may be solved using the 
public domain code LP_SOLVE ${ }^{1}$. The input file is given below:

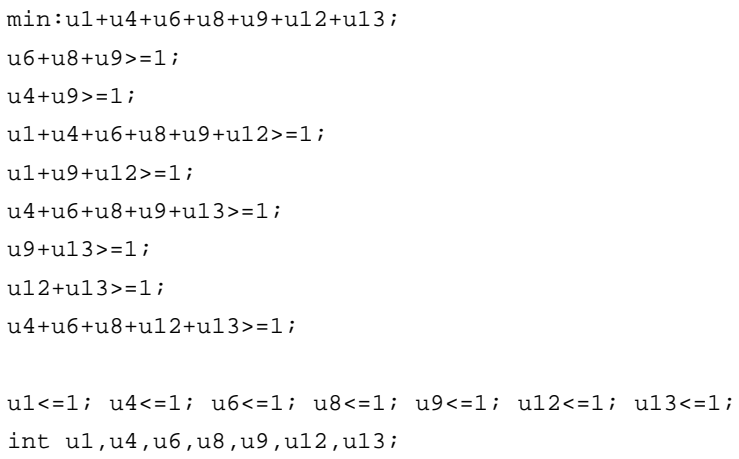

The reader will notice the second group of constraints. As LP_SOLVE can only handle positive values for the variables and taking into account their declaration as integers, the optimisation variables are constrained to be Boolean ( 0 or 1$)$. LP_SOLVE gives the following result: $\{\mathrm{u} 1=0, \mathrm{u} 4=0, \mathrm{u} 6=0, \mathrm{u} 8=0, \mathrm{u} 9=1$, $\mathrm{u} 12=1, \mathrm{u} 13=0\}$ which implies the measurement of the two variables $x 9$ and $x 12$. Analysing the redundancy of the system with these two new measurements, one obtains the following occurrence matrix:

Table 5: occurrence matrix of redundancy equations

\begin{tabular}{|cccccccccc|}
\hline 2 & 3 & 5 & 7 & 9 & 10 & 11 & 12 & 14 & 15 \\
\hline 1 &. & 1 &. & 1 &. &. &. & 1 &. \\
. &. &. &. & 1 & 1 & 1 &. &. &. \\
. & 1 & 1 & 1 &. & 1 &. &. &. &. \\
. &. &. &. &. &. & 1 & 1 & 1 & 1 \\
\hline
\end{tabular}

As the columns corresponding to the variables $x 5$ and $x 11$ are unique, sensor failures measuring these variables are isolable and as variable $x 15$ intervenes in a redundancy equation, the corresponding sensor failure is detectable. The reader may notice that the proposed solution is not unique, in this case, and the measurement of the couple $(x 9, x 13)$ also satisfies the constraints.

\section{CONCLUSION}

In this paper, the problem of sensor network design is addressed using the powerful concept of redundancy degree of variables. The design is formulated as a particular optimisation problem involving Boolean variables and is solved using a mixed binary integer linear programming method. The proposed procedure is developed to handle specifications of importance (expressed by redundancy degrees), measurability of process variables and performances of diagnosis of sensor failures (detectability and isolability). Sensor network design examples illustrate the relevance of the proposed method.

\footnotetext{
1 LP_SOLVE is a public domain code written in $\mathrm{C}$ by $\mathrm{M}$. Berkelaar (michel@es.ele.tue.nl). It can be retrieved from the address ftp://ftp.es.ele.tue.nl/pub/lp_solve
}

\section{REFERENCES}

Ali Y. and Narasimhan S. Sensor network design for maximizing reliability of linear processes. AIChE Journal, 39 (5) 820-828, 1993.

Bagajewicz M.J. Optimal sensor location in process plants. Fith European Symposium on Computer Aided Process Engineering, Escape'95, Bled, Slovenia, June 11-14, 1995.

Carpentier T. and Litwak R. Algorithms and criteria for sensors location in view of supervision. Symposium on Control, Optimization and Supervision, CESA'96, Lille, France, 1996.

Crowe C.M. Test of maximum power for detection of gross errors in process constraints. AIChE Journal, 35 (5) 869-872, 1989.

Deo N. Graph theory with application to engineering and computer sciences. Mc Graw Hill, N.Y., 1967.

Gertler J. and Singer D. Augmented models for statistical fault isolation in complex dynamic systems. American Control Conference, Boston, MA, 1985.

Kretsovalis A. and Mah R.S.H. Effect of redundancy on estimation accuracy in process data reconciliation. Chemical Engineering Sciences, 42, 671-687, 1987.

Madron F. and Veverka V. Optimal selection of measuring points in complex plant by linear models. AIChE Journal, 38 (2) 227-236, 1992.

Mah R.SH. and A.C Tamhane A.C. Detection of gross errors in process data. AIChE Journal, 28 (5) 828-830, 1982.

Maquin D., Fayolle J., Darouach M. and Ragot J. Localization of sensors in large scale industrial systems. Applied Modelling and Simulation of Technological Systems, pp. 33-39, Elsevier, 1987.

Maquin D. and Ragot J. Comparison of gross errors detection methods in process data. 30th IEEE Conference on Decision and Control, Brighton, England, December 11-13, 1991.

Maquin D., Luong M. and Ragot J. About the design of measurement systems and fault accomodation. Engineering Simulation, 13, 1009-1024, 1996.

Narasimhan S. and Mah R.S.H. Generalized likelihood ratio method for gross error identification. AIChE Journal, 33 (9) 1514-1521, 1987.

Ragot J., Darouach M., Maquin D. and Bloch G. Validation de données et diagnostic. Traité des Nouvelles Technologies, série diagnostic et maintenance, Hermès, 1990.

Rosenberg J., Mah R.S.H. and Iordache C. Evaluation of schemes for detecting and identifying gross errors in process data. $I E C$ Research, 26 (3) 555-564, 1987.

Serth J.E. and Heenan W.A. Gross error detection and data reconciliation in steam-metering system. AIChE Journal, 32 (5) 733-742, 1986.

Vaclavek V. and Loucka M. Selection of measurements necessary to achieve multicomponent mass balances in chemical plants. Chemical Engineering Sciences, 31, 1199-1205, 1976. 